\title{
In silico toxicological analyzes of selected toxic compounds from dumpsite or contaminated soils on human health
}

\author{
Omodele Ibraheem ${ }^{1, 凶}$, Toluwase Hezekiah Fatoki ${ }^{1}$, Jesupemi Mercy Enibukun ${ }^{2}$, \\ Bolanle Christianah Faleye ${ }^{3}$ and Daniel Uwaremhevho Momodu ${ }^{4}$ \\ ${ }^{1}$ Department of Biochemistry, Federal University Oye-Ekiti, PMB 373, Oye-Ekiti, Ekiti State, Nigeria \\ ${ }^{2}$ Department of Microbiology, Federal University of Technology, PMB 704 Akure, Nigeria \\ ${ }^{3}$ Department of Chemical Science, Joseph Ayo Babalola University, Ikeji-Arakeji, PMB 5006, Ilesa Osun State, Nigeria \\ ${ }^{4}$ Department of Industrial Chemistry, Federal University Oye-Ekiti, PMB 373, Oye-Ekiti, Ekiti State, Nigeria
}

\section{Article info}

Article history:

Received: $17^{\text {th }}$ July 2019

Accepted: $30^{\text {th }}$ September 2019

\section{Keywords:}

Gene expression network

Molecular docking

Toxicants

Toxicokinetic

Transcription factors

\begin{abstract}
The soil is a key component of natural ecosystems because environmental sustainability depends largely on a sustainable soil ecosystem. The objective of this study was to predict the impact of selected toxic compounds from dumpsite or contaminated soils on human health at the molecular level of biological processes. The in silico methods that were used include toxicokinetics and target gene prediction, molecular docking, and gene expressing network analysis. The result showed bisphenol A (BPA), 2,20-bis(p-chlorophenyl)-1,1-dichloroethane (DDD), 2,20-bis( $p$-chlorophenyl)-1,1-trichloroethane (DDT), diethylhexyl phthalate (DEHP), nonylphenol (NP) and tetrachlorodibenzodioxin (TCDD) as the active toxic compounds that can modulate biological system and are considered as potential cause of several diseases including cancer. The principal target genes include substance-P receptor (also known as Neurokinin 1 receptor), 5-hydroxytryptamine receptor, human serotonin transporter; estrogen receptor alpha; and aryl hydrocarbon receptor. These genes implicated SUZ12, STAT3, and TRIM28 as the major transcription factors while mitogen-activated protein kinases and cyclin-dependent kinases were the major kinases from the proteinprotein interaction. All the six toxicants investigated showed good free binding energies $(\Delta G)$ which were below $-5.0 \mathrm{kcal}^{\mathrm{mol}}{ }^{-1}$. These toxic compounds showed ligand efficiency greater than $0.25 \mathrm{kcal}^{\mathrm{mol}}{ }^{-1}$. HA and would possibly cause fatal damage on human health. The order of in silico predicted toxicity of these compounds were $\mathrm{BPA}>\mathrm{DDD}=\mathrm{DDT}>\mathrm{TCDD}>\mathrm{NP}>\mathrm{DEHP}$. Our results identified potential threats, which the selected toxicants can pose to public health. More importantly, it provides basis for investigation of super bugs (microorganisms) that can remediate these toxicants in our environment. Environmental monitoring and modern wastes management system should be implemented and enforced in the affected countries in order to safeguard the health of the citizenry.
\end{abstract}

(C) University of SS. Cyril and Methodius in Trnava

\section{Introduction}

The soil is a key component of natural ecosystems because environmental sustainability depends largely on a sustainable soil ecosystem (Lombi et al. 1998). The biogeochemical cycles of contaminants have been greatly accelerated by human activities. Typical contaminated sites 
may be classified as either potential or practical, which could be quantitatively described as suspected to cause or constitute harm to human health and the environment - biotic and abiotic processes respectively. The qualitative and quantitative description of potentially contaminated and contaminated sites have been reported (WHO 2013).

The study on soil samples of an animal burial site showed to be characterized by acute toxicity of decayed matter while the area of open waste dumping was found the most dangerous based on the amount of the contaminants (Pasko and Mochalova 2014). Typical study has shown that more industrialized countries had higher bisphenol A (BPA) concentrations in landfill leachate than less industrialized countries (Teuten et al. 2009). Cases of environmental pollution and contamination in developing and developed countries have been reported (Egboka et al. 1989; SCU 2013). Dumpsite has been huge challenge to the global health, because it serves as habitat for carcinogenic chemicals and pathogenic microbes (Odeyemi et al. 2011; Eze and Amaeze 2016). Dumpsites serve as lead for marine and coastal pollution and will account for $8-10 \%$ of anthropogenic greenhouse gas emission in 2025 (ISWA 2016).

Soil microorganisms often serve as the catalysts or promoters of reactions in the subsurface. However, the degradation of any contaminant depends on geochemical conditions and on the presence of microorganisms that are capable of adaptation (Boulding and Barcelona 1991). Uptake of contaminants by organisms occurs by a variety of pathways, most commonly inhalation, dermal sorption and ingestion. Contaminant transfer to organisms may occur by any of these routes, and the major transport route will vary according to the organism and the physicochemical properties of the contaminant (Teuten et al. 2009). Likelihood that health effects will occur from any exposure to a contaminant depends on the toxicity of the contaminant, which can be determined through understanding of: how harmful, how much, how long and how often the exposure occurs. However, differences in the health status, age, diet, gender, family traits and lifestyle will also affect the outcome of the level of exposure to a particular contaminant (Shayler et al. 2009).

The protection of human health has utilized biomonitoring as a vital tool to track and assess the level of exposure of the community to environmental pollutants as well provides measures for local and global health policies (SCU 2013). Characterization of a hazardous waste site provides the understanding to predict future site behavior based on past site behavior (Mercer and Spalding 1991). A basic assumption in performing remediation is that one cannot remediate what is not observed. Consequently, an understanding of what to observe and how to go about making the observations is of utmost importance (Boulding and Barcelona 1991).

Almost all typical research of soil quality often carried out solely on the basis of chemical analyses at the oversight of the resulting effect of the level of toxicity on the organism within the ecosystem. Toxicokinetics is the kinetics of toxicant absorption, distribution, metabolism and excretion (ADME). The investigation of ADME profiling and toxicological (ADME/Tox) screenings during biomonitoring and remediation processes are therefore very important. In silico prediction of toxicokinetics and impact on biomolecules will serve as an additional assessment tool for screening toxic level of soil and foster understanding of impact of pollutants in the environment considering the huge cost and time involve in bioanalytical testing as well as increasing number of toxicants nowadays. In silico prediction of key biomolecular targets of the potential toxic compound will help to ascertain the limit and possible outcome of high exposure. The understanding will help in creating public awareness that is grounded of scientific propositions. The objective of this study was to predict the potential impact of selected toxic compounds from dumpsite or contaminated soils on human health at the molecular level of biological processes.

\section{Experimental}

In silico preparation of toxic ligands

Among the several known toxicants found in typical dumpsites or contaminated soils from 
Table 1. List of 13 selected toxicants among severally found in dumpsites or contaminated soils, which have been implicated in human chronic ailments, among which 6 toxicants (in bold) showed significant predicted targets.

\begin{tabular}{|c|c|c|}
\hline $\begin{array}{l}\text { Serial } \\
\text { No. }\end{array}$ & Type of contaminants & SMILES format \\
\hline 1 & Bisphenol A (BPA) & $\mathrm{CC}(\mathrm{C})(\mathrm{C} 1=\mathrm{CC}=\mathrm{C}(\mathrm{C}=\mathrm{C} 1) \mathrm{O}) \mathrm{C} 2=\mathrm{CC}=\mathrm{C}(\mathrm{C}=\mathrm{C} 2) \mathrm{O}$ \\
\hline 2 & $\begin{array}{l}\text { 2,20-bis(p-chlorophenyl)-1,1- } \\
\text { dichloroethane (DDD) }\end{array}$ & $\mathrm{C} 1=\mathrm{CC}(=\mathrm{CC}=\mathrm{C} 1 \mathrm{C}(\mathrm{C} 2=\mathrm{CC}=\mathrm{C}(\mathrm{C}=\mathrm{C} 2) \mathrm{Cl}) \mathrm{C}(\mathrm{Cl}) \mathrm{Cl}) \mathrm{Cl}$ \\
\hline 3 & $\begin{array}{l}\text { 2,20-bis( } p \text {-chlorophenyl)-1,1- } \\
\text { dichloroethylene (DDE) }\end{array}$ & $\mathrm{C} 1=\mathrm{CC}(=\mathrm{CC}=\mathrm{C} 1 \mathrm{C}(=\mathrm{C}(\mathrm{Cl}) \mathrm{Cl}) \mathrm{C} 2=\mathrm{CC}=\mathrm{C}(\mathrm{C}=\mathrm{C} 2) \mathrm{Cl}) \mathrm{Cl}$ \\
\hline 4 & $\begin{array}{l}\text { 2,20-bis(p-chlorophenyl)-1,1- } \\
\text { trichloroethane (DDT) }\end{array}$ & $\mathrm{C} 1=\mathrm{CC}(=\mathrm{CC}=\mathrm{C} 1 \mathrm{C}(\mathrm{C} 2=\mathrm{CC}=\mathrm{C}(\mathrm{C}=\mathrm{C} 2) \mathrm{Cl}) \mathrm{C}(\mathrm{Cl})(\mathrm{Cl}) \mathrm{Cl}) \mathrm{Cl}$ \\
\hline 5 & diethylhexyl phthalate (DEHP) & $\mathrm{CCCCC}(\mathrm{CC}) \mathrm{COC}(=\mathrm{O}) \mathrm{C} 1=\mathrm{CC}=\mathrm{CC}=\mathrm{C} 1 \mathrm{C}(=\mathrm{O}) \mathrm{OCC}(\mathrm{CC}) \mathrm{CCCC}$ \\
\hline 6 & Hexachlorocyclohexane (HCH) & $\mathrm{C} 1(\mathrm{C}(\mathrm{C}(\mathrm{C}(\mathrm{C}(\mathrm{C} 1 \mathrm{Cl}) \mathrm{Cl}) \mathrm{Cl}) \mathrm{Cl}) \mathrm{Cl}) \mathrm{Cl}$ \\
\hline 7 & Octylphenol (OP) & $\mathrm{CCCCCCCCCC} 1=\mathrm{CC}=\mathrm{C}(\mathrm{C}=\mathrm{C} 1) \mathrm{O}$ \\
\hline 8 & Perfluorooctanesulfonic acid (PFOS) & $\mathrm{C}(\mathrm{C}(\mathrm{C}(\mathrm{C}(\mathrm{C}(\mathrm{F})(\mathrm{F}) \mathrm{S}(=\mathrm{O})(=\mathrm{O}) \mathrm{O})(\mathrm{F}) \mathrm{F})(\mathrm{F}) \mathrm{F})(\mathrm{F}) \mathrm{F})(\mathrm{C}(\mathrm{C}(\mathrm{C}(\mathrm{F})(\mathrm{F}) \mathrm{F})(\mathrm{F}) \mathrm{F})(\mathrm{F}) \mathrm{F})(\mathrm{F}) \mathrm{F}$ \\
\hline 9 & Nonylphenol (NP) & $\mathrm{CCCCCCCCCC} 1=\mathrm{CC}=\mathrm{C}(\mathrm{C}=\mathrm{C} 1) \mathrm{O}$ \\
\hline 10 & Perfluorooctanoic acid (PFOA) & $\mathrm{C}(=\mathrm{O})(\mathrm{C}(\mathrm{C}(\mathrm{C}(\mathrm{C}(\mathrm{C}(\mathrm{C}(\mathrm{C}(\mathrm{F})(\mathrm{F}) \mathrm{F})(\mathrm{F}) \mathrm{F})(\mathrm{F}) \mathrm{F})(\mathrm{F}) \mathrm{F})(\mathrm{F}) \mathrm{F})(\mathrm{F}) \mathrm{F})(\mathrm{F}) \mathrm{F}) \mathrm{O}$ \\
\hline 11 & Trinonylphenylphosphine (TNPP) & $\mathrm{CCCCCCCCC}[\mathrm{P}+](\mathrm{CCCCCCCCC})(\mathrm{CCCCCCCCC}) \mathrm{C} 1=\mathrm{CC}=\mathrm{CC}=\mathrm{C} 1$ \\
\hline 12 & Tetrachlorodibenzodioxin (TCDD) & $\mathrm{C} 1=\mathrm{C} 2 \mathrm{C}(=\mathrm{CC}(=\mathrm{C} 1 \mathrm{Cl}) \mathrm{Cl}) \mathrm{OC} 3=\mathrm{CC}(=\mathrm{C}(\mathrm{C}=\mathrm{C} 3 \mathrm{O} 2) \mathrm{Cl}) \mathrm{Cl}$ \\
\hline 13 & Toxaphene (SONATOX) & $\mathrm{C} 1 \mathrm{C} 2 \mathrm{C}(\mathrm{C}(\mathrm{C}(\mathrm{C} 1(\mathrm{Cl}) \mathrm{Cl})(\mathrm{C} 2(\mathrm{CCl}) \mathrm{CCl}) \mathrm{CCl}) \mathrm{Cl}) \mathrm{Cl}$ \\
\hline
\end{tabular}

literatures (Teuten et al. 2009; Valentin et al. 2013; Yao et al. 2015), 13 toxicants (Table 1), which were known to be critically implicated in implicated in human chronic ailments such as cancer, chronic cough, and neurological disorder, were selected and used for this study. Available structures of these compounds were obtained from the PubChem Compound Database in structure data file (sdf) and canonical Simplified Molecular Input Line Entry Specification format (SMILES). All file conversion to protein data bank (pdb) format were performed using PyMol v2.0.7.

\section{In silico targets prediction and toxicokinetics}

In silico targets prediction for the toxicants were done on SwissTargetPrediction server, where Homo sapiens was selected as target organism (Diana et al. 2019). Among the 13 toxicants, 6 toxicants showed significant predicted targets (i.e. active ligands; Table 1), and these were then subjected to in silico Absorption-DistributionMetabolism-Excretion (ADME) screening on SwissADME server (Diana et al. 2017). ADME screening was performed at default parameters.

\section{Molecular docking}

The molecular docking studies for the 6 toxicants against the 6 targets were carried out according to the method described by Fatoki et al. (2018a). The three-dimension (3D) structures of selected 6 targets were then obtained from RCSB Protein Data Bank (PDB). Briefly, all water molecules, hetero atoms, and multichains were removed from the crystal structure of the prepared targets using PyMol v2.0.7. The Gasteiger partial charges were added to the ligand atoms prior to docking. The docking parameter of each prepared ligand and each prepared target, were setup using AutoDock Tools (ADT) v1.5.6 (Morris et al. 2009) and saves the output file in pdbqt format. Molecular docking program AutoDock Vina v1.1.2 (Trott and Olson 2010) was employed to perform the docking experiment from the command line. After docking, the ligands were analyzed and visualized using ADT and PyMol v2.0.7. The ligand efficiency (LE) was evaluated from the equation, $\mathrm{LE}=-\Delta G / \mathrm{HA}$, where $\Delta G$ is the free energy of binding and HA is the number heavy atoms (non-hydrogen atoms) of the ligand (Padmanabhan et al. 2016).

\section{Target gene expression analyses}

The upstream regulatory networks from signatures of differentially expressed genes obtained from toxicants target prediction, were determined by 
Nova Biotechnol Chim (2019) 18(2): 144-153

Table 2. Predicted targets of the dumpsites/contaminated soils active toxicants.

\begin{tabular}{|c|c|c|c|c|c|c|c|c|c|}
\hline \multirow{2}{*}{$\begin{array}{l}\text { Serial } \\
\text { No. }\end{array}$} & \multirow{2}{*}{ Targets } & \multirow{2}{*}{ Gene name } & \multirow{2}{*}{ UniProt ID } & \multicolumn{4}{|c|}{ Dumpsite toxicants } & \multirow[b]{2}{*}{$\mathbf{E}$} & \multirow[b]{2}{*}{$\mathbf{F}$} \\
\hline & & & & $\mathbf{A}$ & B & $\mathbf{C}$ & D & & \\
\hline 1 & Estrogen receptor & ESR1 & P03372 & $* * * *$ & & & & $* * * *$ & \\
\hline 2 & Estrogen receptor beta (by homology) & ESR2 & Q92731 & $* * * *$ & & & & & \\
\hline 3 & 5-hydroxytryptamine receptor 6 & HTR6 & P50406 & $* * * *$ & $* * *$ & $* * *$ & & & \\
\hline 4 & FAD-linked sulfhydryl oxidase ALR & GFER & P55789 & $* * *$ & & & & & \\
\hline 5 & $\begin{array}{l}\text { Carbonic anhydrase } 1,2,3,5 \mathrm{~A}, 5 \mathrm{~B}, \\
7,13\end{array}$ & $\begin{array}{l}\text { CA1, CA2, CA3, } \\
\text { CA5A, CA5B, } \\
\text { CA7, CA13 }\end{array}$ & $\begin{array}{l}\text { P00915, P00918, } \\
\text { P07451, P35218, } \\
\text { Q9Y2D0, } \\
\text { P43166, Q8N1Q1 }\end{array}$ & $* * *$ & & & & & \\
\hline 6 & Androgen receptor (by homology) & $\mathrm{AR}$ & P10275 & $* * *$ & & & $* *$ & & \\
\hline 7 & Arachidonate lipoxygenase & $\begin{array}{l}\text { ALOX5 } \\
\text { ALOX12, } \\
\text { ALOX15 }\end{array}$ & $\begin{array}{l}\text { P09917, P18054, } \\
\text { P16050 }\end{array}$ & $* * *$ & & & & & \\
\hline 8 & Alpha-2A adrenergic receptor & ADRA2A & P08913 & & $* * *$ & & & & \\
\hline 9 & Microtubule-associated protein tau & MAPT & P10636 & & $* * *$ & $* *$ & $* *$ & & \\
\hline 10 & $\begin{array}{l}\text { Sodium-dependent noradrenaline } \\
\text { transporter }\end{array}$ & SLC6A2 & P23975 & & $* * *$ & $* * *$ & & $* * * *$ & \\
\hline 11 & $\begin{array}{l}\text { Sodium-dependent dopamine } \\
\text { transporter }\end{array}$ & SLC6A3 & Q01959 & & $* * *$ & $* * *$ & & & \\
\hline 12 & $\begin{array}{l}\text { Sodium-dependent serotonin } \\
\text { transporter }\end{array}$ & SLC6A4 & P31645 & & $* * *$ & $* * *$ & & $* * * *$ & \\
\hline 13 & $\begin{array}{l}\text { Sodium-dependent proline transporter } \\
\text { (by homology) }\end{array}$ & SLC6A7 & Q99884 & & $* * *$ & $* * *$ & & & \\
\hline 14 & $\begin{array}{l}\text { Sodium- and chloride-dependent } \\
\text { glycine transporter } 1 \text { (by homology) }\end{array}$ & SLC6A9 & P48067 & & $* * *$ & $* * *$ & & & \\
\hline 15 & $\begin{array}{l}\text { Sodium- and chloride-dependent } \\
\text { neutral and basic amino acid } \\
\text { transporter } \mathrm{B}(0+) \text { (by homology) }\end{array}$ & SLC6A14 & Q9UN76 & & $* * *$ & $* * *$ & & & \\
\hline 16 & $\begin{array}{l}\text { 5-hydroxytryptamine receptor } 2 \mathrm{~A}, \\
2 \mathrm{~B}, 2 \mathrm{C} \text { (by homology) }\end{array}$ & $\begin{array}{l}\text { HTR2A, HTR2B } \\
\text { HTR2C, HTR6 }\end{array}$ & $\begin{array}{l}\text { P28223, P41595, } \\
\text { P28335, P50406 }\end{array}$ & & $* * *$ & $* * *$ & & $* * * *$ & \\
\hline 17 & $\begin{array}{l}\text { Adrenergic receptor Alpha-2B, } 2 \mathrm{C} \\
\text { (by homology) }\end{array}$ & $\begin{array}{l}\text { ADRA2B, } \\
\text { ADRA2C }\end{array}$ & P18089, P18825 & & $* * *$ & $* * *$ & & $* * * *$ & \\
\hline 18 & $\begin{array}{l}\text { Adrenergic receptor Beta-1, Beta-2, } \\
\text { Beta-3 }\end{array}$ & $\begin{array}{l}\text { ADRB1, ADRB2, } \\
\text { ADRB3 }\end{array}$ & $\begin{array}{l}\text { P08588, P07550, } \\
\text { P13945 }\end{array}$ & & & & & $* * * *$ & \\
\hline 19 & $\begin{array}{l}\text { Protein kinase } \mathrm{C} \text { alpha type, beta } \\
\text { type, gamma type, theta type, delta } \\
\text { type regulatory subunit }\end{array}$ & $\begin{array}{l}\text { PRKCA, PRKCB, } \\
\text { PRKCG, PRKCQ, } \\
\text { PRKCD }\end{array}$ & $\begin{array}{l}\text { P17252, P05771, } \\
\text { P05129, Q04759, } \\
\text { Q05655 }\end{array}$ & & & & $* *$ & & \\
\hline 20 & $\begin{array}{l}\text { Tyrosine-protein phosphatase non- } \\
\text { receptor type } 1 \text {, type } 2\end{array}$ & PTPN1, PTPN2 & P18031, P17706 & & & & $* *$ & & \\
\hline 21 & $\begin{array}{l}\text { Opioid receptor Mu-type, Delta-type, } \\
\text { Kappa-type }\end{array}$ & $\begin{array}{l}\text { OPRM1, OPRD1, } \\
\text { OPRK1 }\end{array}$ & $\begin{array}{l}\text { P35372, P41143, } \\
\text { P41145 }\end{array}$ & & & & $* *$ & & \\
\hline 22 & Adenosine receptor A3 & ADORA3 & P33765 & & & & & $* * * *$ & \\
\hline 23 & Substance-P receptor, $\mathrm{K}$ receptor & TACR1, TACR2 & $\mathrm{P} 25103, \mathrm{P} 21452$ & & & & & $* * * *$ & \\
\hline 24 & $\begin{array}{l}\text { Testis-specific androgen-binding } \\
\text { protein }\end{array}$ & SHBG & P04278 & & & & & $* * * *$ & \\
\hline 25 & $\begin{array}{l}\text { Vascular endothelial growth factor } \\
\text { receptor } 1,2,3\end{array}$ & $\begin{array}{l}\text { FLT1, KDR, } \\
\text { FLT4 }\end{array}$ & $\begin{array}{l}\text { P17948, P35968, } \\
\text { P35916 }\end{array}$ & & & & & & $* * * *$ \\
\hline 26 & Aryl hydrocarbon receptor & AHR & P35869 & & & & & & $* * * *$ \\
\hline
\end{tabular}

$*(20-40 \%), * *(40-60 \%), * * *(60-80 \%)$, **** $(80-100 \%)$ probability of binding on target. Probabilities have been computed based on a cross-validation. They may therefore not represent the actual probability of success for any new molecule.
$(\mathrm{A})=$ Bisphenol A $(\mathrm{BPA})$
(B) $=2,20$-bis ( $p$-chlorophenyl)-1,1-dichloroethane (DDD)
(C) $=2,20$-bis ( $p$-chlorophenyl)-1,1-trichloroethane (DDT)
(D) $=$ Diethylhexyl phthalate (DEHP)
(E) = Nonylphenol (NP)
$(\mathrm{F})=$ Tetrachlorodibenzodioxin $(\mathrm{TCDD})$

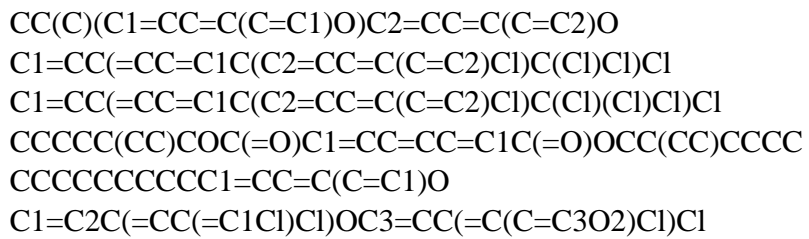


transcription factor enrichment analysis, proteinprotein interaction network expansion and kinase enrichment analysis, using the 50 predicted target genes on eXpression2Kinases (X2K) webserver (Clarke et al. 2018).

\section{Results and Discussion}

\section{Dumpsite/contaminated soil active toxicants and their predicted targets}

In this study, six toxicants from typical dumpsite or contaminated soil were found active in biological system at percentage probability greater than $20 \%$ (Table 2), although octylphenol (data not shown) was also found to be about $20 \%$ less active than nonylphenol. Fifty predicted targets (using gene name or UniProt ID) were obtained (Table 2) of which six were selected for further investigation based on their crucial implication on human health (Table 3). These were classified mainly into: (1) membrane proteins, such as substance-P receptor (also known as Neurokinin 1 receptor), 5-hydroxytryptamine receptor and human serotonin transporter; (2) nuclear proteins which include estrogen receptor alpha; and (3) transcription proteins which include aryl hydrocarbon receptor. The serotonin $2 \mathrm{~A}$ receptor (5-HT2AR) is associated with diseases, such as bipolar disorder, depression, schizophrenia,
Alzheimer's disease and Parkinson's disease (Aznar and Hervig 2016). Structural elucidation has shown that 5-HT2AR possesses a unique sideextended cavity near the orthosteric binding site and possibly contributes to the high selectivity of ligands such pimavanserin (Kimura et al. 2019). Estrogen receptor (ER), an allosteric signaling protein and example of nuclear receptors, is a well validated therapeutic target for the treatment of estrogen receptor positive breast cancer (Burks et al. 2017; Fatoki et al. 2018a). Study on model compounds that targets ER has provided insight on the close structural similarity with tamoxifen, the FDA-approved drug which is a potent selective estrogen receptor modulator (Fatoki et al. 2018a). TCDD (2,3,7,8-tetrachlorodibenzo- $p$-dioxin) is the best known high-affinity ligand for aryl hydrocarbon receptor (AHR), and mediates its toxicity via activation of AHR. Increased levels of AHR and its target genes have been implicated in several belligerent tumors such as skin tumors, glioblastoma, or non-small cell lung cancer (Schulte et al. 2017).

\section{Predicted toxicokinetic properties of the toxicants}

The ADME parameter of the selected six toxicants (Table 3) showed that bisphenol A (BPA) and nonylphenol (NP) were soluble and moderately soluble (respectively), and both likely have high

Table 3. Predicted toxicokinetic parameters of the dumpsites/contaminated soils active toxicants.

\begin{tabular}{|c|c|c|c|c|c|c|c|}
\hline \multirow{2}{*}{$\begin{array}{l}\text { Serial } \\
\text { No. }\end{array}$} & \multirow[t]{2}{*}{ Parameters } & \multicolumn{6}{|c|}{ Selected dumpsite toxicants } \\
\hline & & BPA & DDD & DDT & DEHP & NP & TCDD \\
\hline 1 & Molecular Weight & 228.29 & 320.04 & 354.49 & 390.56 & 220.35 & 321.97 \\
\hline 2 & Heavy Atoms (HA) & 17 & 18 & 19 & 28 & 16 & 18 \\
\hline 3 & Molar Refractivity & 69.44 & 80.31 & 85.15 & 116.3 & 71.89 & 73.07 \\
\hline 4 & Total Polar Surface Area $\left(A^{2}\right)$ & 40.46 & 0 & 0 & 52.60 & 20.23 & 18.46 \\
\hline 5 & Consensus LogP & 3.6 & 5.44 & 5.89 & 6.17 & 4.51 & 5.29 \\
\hline 6 & ESOL Class & Soluble & $\begin{array}{l}\text { Moderately } \\
\text { soluble }\end{array}$ & $\begin{array}{l}\text { Poorly } \\
\text { soluble }\end{array}$ & $\begin{array}{l}\text { Poorly } \\
\text { soluble }\end{array}$ & $\begin{array}{l}\text { Moderately } \\
\text { soluble }\end{array}$ & $\begin{array}{l}\text { Poorly } \\
\text { soluble }\end{array}$ \\
\hline 7 & Gastrointestinal Absorption & High & Low & Low & High & High & Low \\
\hline 8 & Blood Brain Barrier (BBB) Permeant & Yes & No & No & No & Yes & No \\
\hline 9 & P-glycoprotein Substrate & No & No & No & Yes & No & Yes \\
\hline 10 & Cytochrome P450 Inhibitor & $\begin{array}{l}\text { CYP1A2, } \\
\text { CYP2D6 }\end{array}$ & $\begin{array}{l}\text { CYP1A2, } \\
\text { CTP2C9, } \\
\text { CYP2C19, } \\
\text { CYP2D6 }\end{array}$ & $\begin{array}{l}\text { СТР2C9, } \\
\text { СYР2C19 }\end{array}$ & $\begin{array}{l}\text { СТР2C9, } \\
\text { СYР3A4 }\end{array}$ & $\begin{array}{l}\text { CYP1A2, } \\
\text { CYP2C19, } \\
\text { CYP2D6 }\end{array}$ & CYP2C9 \\
\hline 11 & Skin permeation $\log K_{p}\left(\mathrm{~cm} \cdot \mathrm{s}^{-1}\right)$ & -5.34 & -3.98 & -3.56 & -3.39 & -3.55 & -3.44 \\
\hline 12 & Lipinski Violation & 0 & 1 & 1 & 1 & 0 & 1 \\
\hline 13 & Bioavailability Score & 0.55 & 0.55 & 0.55 & 0.55 & 0.55 & 0.55 \\
\hline 14 & Synthetic Accessibility & 1.43 & 2.37 & 2.37 & 4.12 & 1.63 & 2.71 \\
\hline
\end{tabular}


Table 4. Docking parameters implemented in AutoDockTool for selected 6 targets.

\begin{tabular}{lllll}
\hline $\begin{array}{l}\text { Serial } \\
\text { No. }\end{array}$ & Selected Target & $\begin{array}{l}\text { Center grid box } \\
\text { [points] }\end{array}$ & $\begin{array}{l}\text { Size } \\
{[\text { points] }}\end{array}$ & $\begin{array}{c}\text { Spacing } \\
{[\AA]}\end{array}$ \\
\hline 1 & $\begin{array}{l}\text { Adrenergic receptor alpha-2A } \\
\text { (PDB ID: 5UIG, Chain A) }\end{array}$ & $171.339 \times 21.415 \times 21.578$ & $126 \times 108 \times 112$ & 0.675 \\
2 & $\begin{array}{l}\text { Aryl hydrocarbon receptor } \\
\text { (PDB ID: 5NJ8, Chain A) }\end{array}$ & $-8.185 \times 37.203 \times 215.837$ & $126 \times 126 \times 126$ & 0.375 \\
3 & $\begin{array}{l}\text { Estrogen receptor } \\
\text { (PDB ID: 5T97, Chain A) }\end{array}$ & $-33.601 \times 18.875 \times-21.737$ & $120 \times 126 \times 122$ & 0.375 \\
4 & $\begin{array}{l}\text { Sodium-dependent serotonin transporter } \\
\text { (PDB ID: 5I6Z, Chain A) }\end{array}$ & $36.842 \times 183.210 \times 142.913$ & $126 \times 100 \times 126$ & 0.475 \\
5 & $\begin{array}{l}\text { Substance-P receptor } \\
\text { (PDB ID: 6HLL, Chain A) }\end{array}$ & $2.367 \times 28.422 \times-26.136$ & $70 \times 80 \times 126$ & 0.875 \\
6 & 5-hydroxytryptamine receptor 2A & $43.501 \times-0.464 \times 60.742$ & $126 \times 66 \times 76$ & 0.775 \\
\hline
\end{tabular}

gastrointestinal absorption (GA), may permeate blood-brain barrier (BBB) and could inhibit cytochrome P450 (CYP1A2 and CYP2D6). On the other hand, diethylhexyl phthalate (DEHP) and tetrachlorodibenzodioxin (TCDD) were identified to possibly have high and low GA respectively, and both are poorly soluble, may not permeate blood-brain barrier (BBB), and serve as $\mathrm{P}$-glycoprotein substrates as well as inhibitors of cytochrome P450 (CYP2C9).

The best bioavailability score and synthetic accessibility (SA) score is 1.0 which is an indication of the amount of the compound that could reach the active site and extent of ease of synthesis of the compound, respectively (Diana et al. 2017; Fatoki et al. 2018b). The SA of the toxicants in this study ranged between 1.43 and 4.12, and showed that they were from or could make up synthetic materials. Usually, not all contaminant found in soil are biologically available. Bioavailability has been described as the physicochemical access that a toxicant has to the biological processes of an organism (Allen 2002). The bioavailability of a contaminant depends on the characteristics of the soil and of the site (Shayler et al. 2009).

\section{Binding energy and efficiency of the toxicants}

The parameter for docking analysis and predicted binding site amino acid residues are shown in Table 4. All the six toxicants investigated in this study show good free binding energies below $-5.0 \mathrm{kcal}^{\mathrm{mol}}{ }^{-1}$ as shown in Table 5. DDT and BPA were found to have highest predicted free binding energy in four and two of the targets respectively. The interaction of some of the ligands with the protein targets are shown in Fig. 1.

The ligand efficiency (LE) is a useful metric to assess binding affinity of compounds with respect to the number of non-hydrogen atoms (Schultes et al. 2010). The toxic compounds showing LE greater than $0.25 \mathrm{kcal}_{\mathrm{mol}}{ }^{-1}$. HA will possibly cause fatal damage on human health. Based on the ligand efficiency result (Table 6), the order of toxicity of the compounds investigated in this study is BPA > $\mathrm{DDD}=\mathrm{DDT}>\mathrm{TCDD}>\mathrm{NP}>$ DEHP.

Table 5. Docking score for the binding free energy between the selected 6 targets and 6 active toxicants.

\begin{tabular}{|c|c|c|c|c|c|c|c|}
\hline \multirow{2}{*}{$\begin{array}{l}\text { Serial } \\
\text { No. }\end{array}$} & \multirow{2}{*}{ Selected Target } & \multicolumn{6}{|c|}{ Binding energy $\left[\mathrm{kcal}^{\left.-\mathrm{mol}^{-1}\right]}\right.$ of the selected toxicants } \\
\hline & & BPA & DDD & DDT & DEHP & NP & TCDD \\
\hline 1 & Estrogen receptor (PDB ID: 5T97) & -9.1 & -8.5 & -7.3 & -7.5 & -6.7 & -7.4 \\
\hline 2 & $\begin{array}{l}\text { Sodium-dependent serotonin transporter (PDB ID: } \\
\text { 5I6Z) }\end{array}$ & -9.5 & -8.2 & -9.8 & -8.2 & -7.5 & -8.2 \\
\hline 3 & Adrenergic receptor alpha-2A (PDB ID: 5UIG) & -11.9 & -11.1 & -9.2 & -7.6 & -6.8 & -10.3 \\
\hline 4 & Aryl hydrocarbon receptor (PDB ID: 5NJ8) & -8.7 & -8.3 & -9.5 & -7.2 & -7.0 & -6.2 \\
\hline 5 & Substance-P receptor (PDB ID: 6HLL) & -9.0 & -8.2 & -9.9 & -7.5 & -7.1 & -9.2 \\
\hline 6 & 5-hydroxytryptamine receptor 2A (PDB ID: 6A93) & -9.2 & -8.6 & -10.5 & -8.3 & -7.8 & -8.8 \\
\hline
\end{tabular}




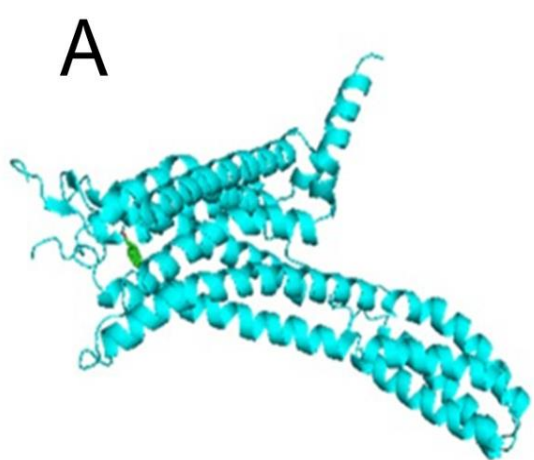

B

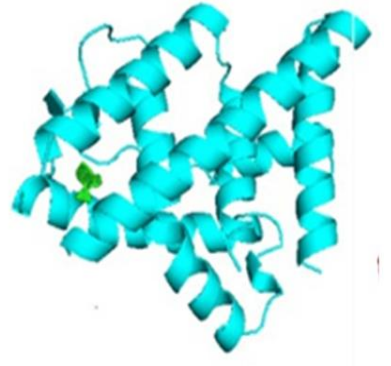

C

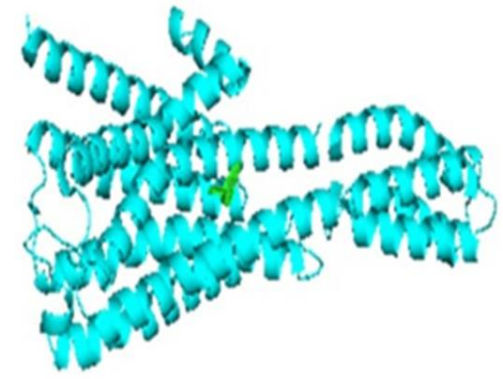

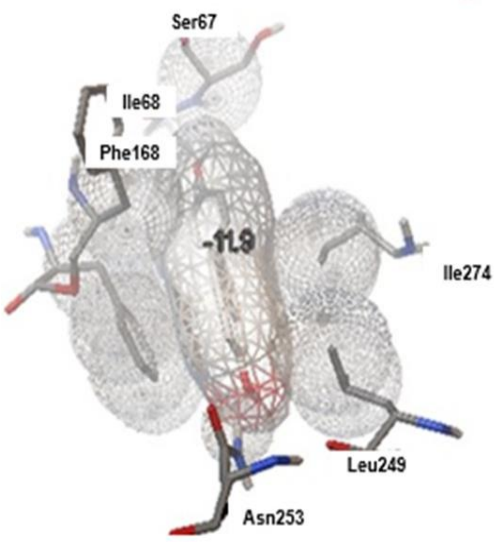
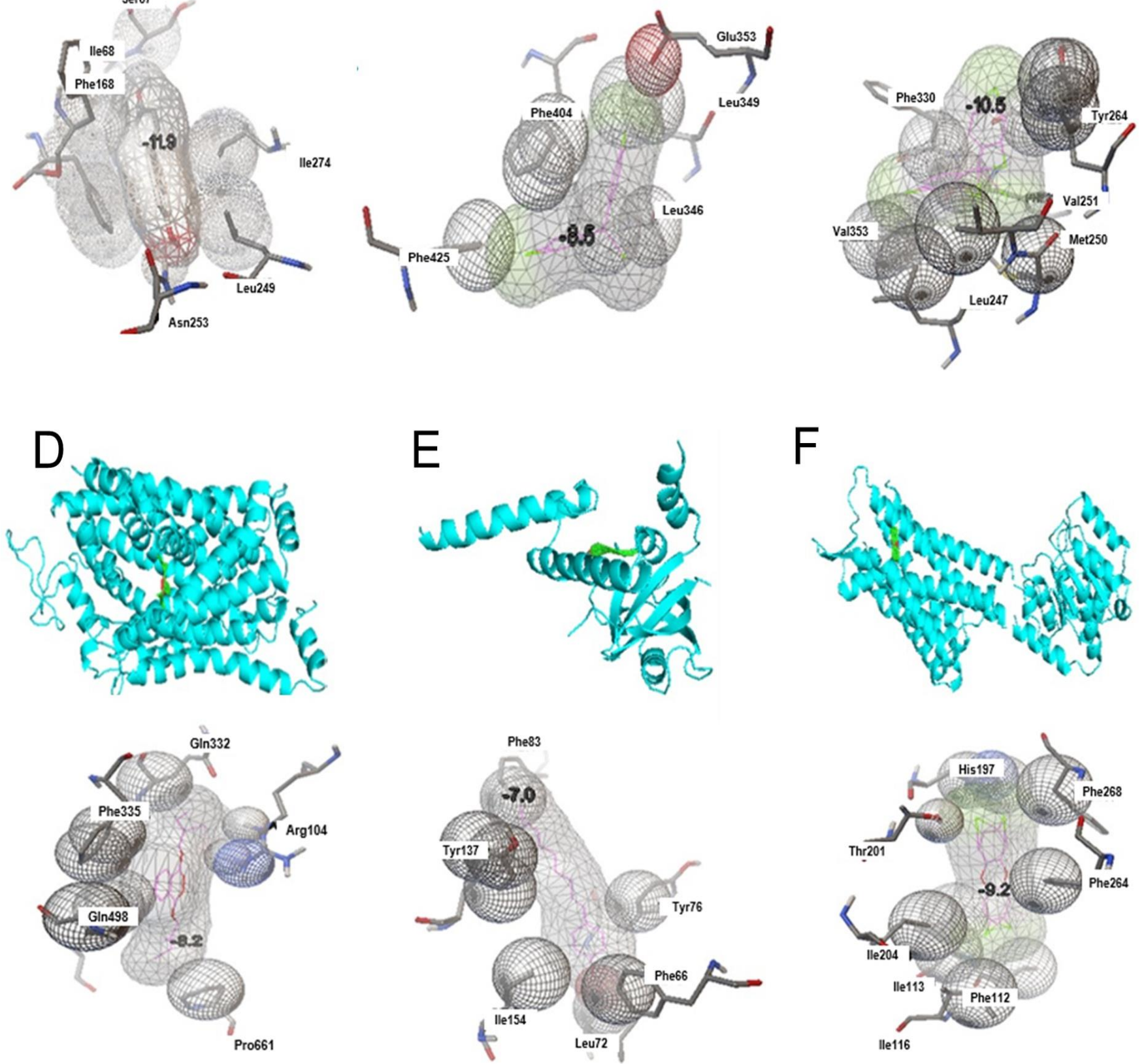

Fig. 1. Binding Pose and Score of the 6 active toxicants, where (A) - BPA interaction with 5UIG (- $\left.11.9 \mathrm{kcal}^{\mathrm{mol}}{ }^{-1}\right)$; (B) - DDD interaction with 5 T97 $\left(-8.5 \mathrm{kcal}^{\left.-\mathrm{mol}^{-1}\right)}\right.$; (C) - DDT interaction with 6A93 $\left(-10.5 \mathrm{kcal}^{\mathrm{mol}} \mathrm{m}^{-1}\right)$; $(\mathbf{D})-\mathrm{DEHP}$ interaction with $5 \mathrm{I} 6 \mathrm{Z}\left(-8.2 \mathrm{kcal}^{\mathrm{mol}}{ }^{-1}\right) ;(\mathbf{E})-\mathrm{NP}$ interaction with $5 \mathrm{NJ} 8\left(-7.0 \mathrm{kcal}^{\mathrm{mol}} \mathrm{mol}^{-1}\right)$ ( F) - TCDD interaction with 6HLL (- $\left.9.2 \mathrm{kcal}_{\mathrm{mol}}{ }^{-1}\right)$ visualized on PyMol and ADT, respectively. 
Table 6. Ligand Efficiencies (LE) of docked scores of selected 6 targets and 6 active toxicants.

\begin{tabular}{|c|c|c|c|c|c|c|c|}
\hline \multirow{2}{*}{$\begin{array}{l}\text { Serial } \\
\text { No. }\end{array}$} & \multirow{2}{*}{ Selected Target } & \multicolumn{6}{|c|}{$\begin{array}{l}\text { Ligand efficiency of the selected toxicants } \\
\left.\text { [kcal.mol }{ }^{-1} \mathrm{HA}\right]\end{array}$} \\
\hline & & BPA & DDD & DDT & DEHP & $\mathbf{N P}$ & TCDD \\
\hline 1 & Estrogen receptor (PDB ID: 5T97) & 0.54 & 0.47 & 0.38 & 0.27 & 0.42 & 0.41 \\
\hline 2 & Sodium-dependent serotonin transporter (PDB ID: 5I6Z) & 0.56 & 0.46 & 0.52 & 0.29 & 0.47 & 0.46 \\
\hline 3 & Adrenergic receptor alpha-2A (PDB ID: 5UIG) & 0.70 & 0.62 & 0.48 & 0.27 & 0.43 & 0.57 \\
\hline 4 & Aryl hydrocarbon receptor (PDB ID: 5NJ8) & 0.51 & 0.46 & 0.50 & 0.26 & 0.44 & 0.34 \\
\hline 5 & Substance-P receptor (PDB ID: 6HLL) & 0.53 & 0.46 & 0.52 & 0.27 & 0.44 & 0.51 \\
\hline 6 & 5-hydroxytryptamine receptor 2A (PDB ID: 6A93) & 0.54 & 0.48 & 0.55 & 0.30 & 0.49 & 0.49 \\
\hline
\end{tabular}

Gene expression network modulated by the toxicants

The overall expression network of the fifty predicted target genes obtained in this study as shown in Fig. 2, highly implicated SUZ12 (polycomb protein) with hypergeometric $p$-value of $5.44 \times 10^{-10}$, as the most enriched transcription factors among others, which are associated with the active toxicants from the typical dumpsite while the mitogen-activated protein kinases (MAPKs) and cyclin-dependent kinases (CDKs) with maximum hypergeometric $p$-value of $2.70 \times 10^{-15}$ were the major kinases from the protein-protein interaction (PPI) as shown in Fig. 2. Differential gene expression study has become one of the imperative methods to discover genes which are essential in diagnosis and prediction of diseases such cancer (Fatoki et al. 2018b). The transcription factors such as TRIM28, SUZ12 and STAT3 are associated with the proliferation of cancer cells. The result of this study was found similar with that of enrichment analysis of urethane-targeted genes which showed TRIM28 and SUZ12 as most

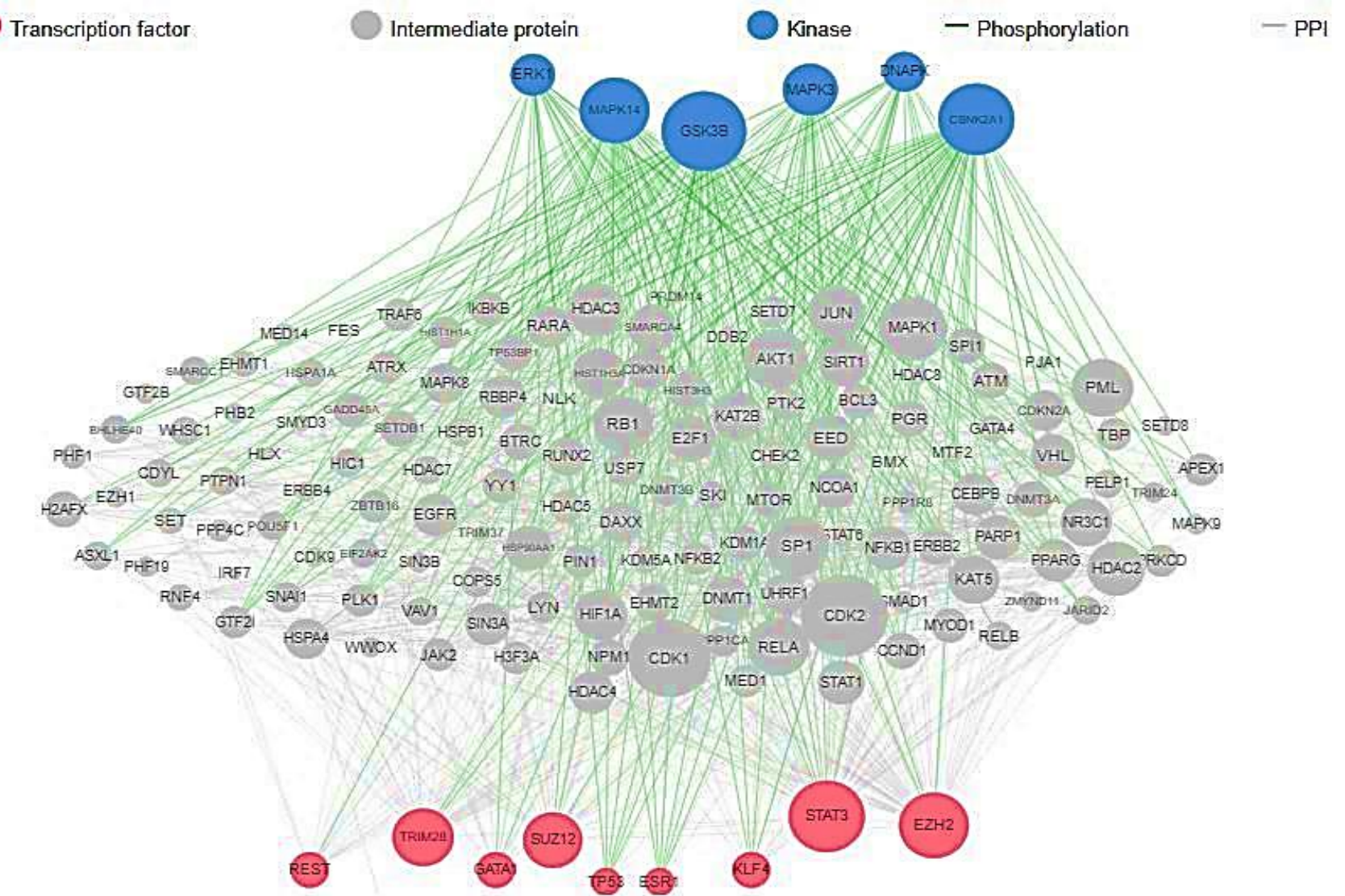

Fig. 2. Overall network of target genes of active toxicants from typical dumpsite or contaminated soil generated by eXpression2Kinases server. 
expressed transcription factors (Fatoki et al. 2018b). However, endometrial stromal tumors may be a result of a chromosomal aberration involving SUZ12.

\section{Conclusions}

This study has unravelled some of the chemical compounds from typical dumpsite or grossly contaminated soil that might actively modulate biological system and are potential cause of several ailments which include cancer, bipolar disorder, schizophrenia, neurodegenerative disease and others. This study has showed the threat which toxicants pose to public health. More importantly, it provides avenue for further investigation of super bugs (microorganisms) that can remediate these toxicants in our environment through bioaugmentation, which improves the biodegradative capacities of contaminated sites. Environmental monitoring and modern wastes management system should be implemented and enforced in order to safeguard the public health and prevent indiscriminate dumping of wastes in the communities and regularize human habitation far away from existing dumpsites.

\section{Conflict of Interest}

The authors declare that they have no conflict of interest.

\section{References}

Allen HE (2002) Bioavailability of metals in terrestrial ecosystems: importance of partitioning for bioavailability to invertebrates, microbes, and plants. SETAC Press. Pensacola, Florida, USA, 158 p.

Aznar S, Hervig ME (2016) The 5-HT2A serotonin receptor in executive function: implications for neuropsychiatric and neurodegenerative diseases. Neurosci. Biobehav. Rev. 64: 63-82.

Boulding JR, Barcelona MJ (1991) Geochemical variability of the natural and contaminated subsurface environment. In Site characterization for subsurface remediation. Center for Environmental Research Information Office of Research and Development U.S. Environmental Protection Agency, Cincinnati, USA, p. 103-122.

Burks HE, Abrams T, Kirby CA, Baird J, Fekete A, Hamann LG, Kim S, Lombardo F, Loo A, Lubicka D, Macchi K, McDonnell DP, Mishina Y, Norris JD, Nunez J, Saran C, Sun Y, Thomsen NM, Wang C, Wang J, Peukert S (2017) Discovery of an acrylic acid based tetrahydroisoquinoline as an orally bioavailable selective estrogen receptor degrader for ER alpha + breast cancer. J. Med. Chem. 60: 2790-2818.

Clarke DJB, Kuleshov MV, Schilder BM, Torre D, Duffy ME, Keenan AB, Lachmann A, Feldmann AS, Gundersen GW, Silverstein MC, Wang Z, Ma'ayan A (2018) eXpression2Kinases (X2K) Web: linking expression signatures to upstream cell signaling networks. Nucleic Acids Res. 46, W1: 171-179.

Diana A, Michielin O, Zoete V (2017) SwissADME: a free web tool to evaluate pharmacokinetics, druglikeness and medicinal chemistry friendliness of small molecules. Sci. Rep. 7: 42717.

Diana A, Michielin O, Zoete V (2019) SwissTargetPrediction: updated data and new features for efficient prediction of protein targets of small molecules. Nucleic Acids Res. 47: W357-W364.

Egboka BC, Enwankwor GI, Orajaka IP, Ejiofor AO (1989) Principles and problems of environmental pollution of groundwater resources with case examples from developing countries. Environ. Health Perspect. 83: 39-68.

Eze CT, Amaeze NH (2016) Microbiological and heavy metal characterization of soil from an open hospital waste dumpsite in Enugu, Nigeria. Asian J. Microbiol. Biotechnol. Environ. Sci. 18: 587-595.

Fatoki TH, Awofisayo OA, Ogunyewo OA, Ugboko HU, Sanni DM (2018a) Impacts of analogy and dimerization of bioactive compounds on molecular biological functions. J. Adv. Med. Pharma. Sci. 19: 1-14.

Fatoki TH, Dutta S, Oyedele AS (2018b) Uncovering the selective drug targets for urethane mediated cancer by network approach. .J Appl. Life Sci. Int. 19: 1-12.

ISWA - International Solid Waste Association (2016) A roadmap for closing waste dumpsites The world's most polluted places. ISWA's Scientific and Technical Committee Work-Program 2015-2016, $124 \mathrm{p}$.

Kimura TK, Asada H, Inoue A, Kadji FMN, Im, D, Mori C, Arakawa T, Hirata K, Nomura Y, Nomura N, Aoki J, Iwata S, Shimamura T (2019) Structures of the 5-HT2A receptor in complex with the antipsychotics risperidone and zotepine. Nat. Struct. Mol. Biol. 26: 121-128.

Lombi E, Wenzel WW, Adriano DC (1998) Soil contamination, risk reduction and remediation. Land Contamin. Reclamat. 6: 183-187.

Mercer JW, Spalding CP (1991) Site characterization overview. In Site Characterization for Subsurface Remediation. Center for Environmental Research Information Office of Research and Development U.S. Environmental Protection Agency, Cincinnati, USA, p. $12-21$

Morris GM, Huey R, Lindstrom W, Sanner MF, Belew RK, Goodsell DS, Olson AJ (2009) AutoDock4 and AutoDockTools4: automated docking with selective receptor flexibility. J. Comput. Chem. 30(16): 2785-2791.

Odeyemi, AT, Faweya EB, Agunbiade OR, Ayeni SK (2011) Bacteriological, mineral and radioactive contents of leachate samples from dumpsite of Ekiti State Government Destitute Centre in Ado-Ekiti. Arch. Appl. 
Sci. Res. 3(4): 92-108.

Padmanabhan B, Ramu M, Mathur S, Unni S, Thiyagarajan S (2016) Identification of new inhibitors for human SIRT1: an in-silico approach. Med. Chem. 12(4): 347-361.

Pasko OA, Mochalova TN (2014) Toxicity assessment of contaminated soils of solid domestic waste landfill. IOP Conf. Ser. Earth Environ. Sci, 21: 012044

Schulte KW, Green E, Wilz A, Platten M, Daumke O (2017) Structural basis for aryl hydrocarbon receptor-mediated gene activation. Structure 25(1-9): e1-e3.

Schultes S, de Graaf C, Haaksma EEJ, de Esch IJP, Leurs R, Krämer O (2010) Ligand efficiency as a guide in fragment hit selection and optimization. Drug Discov. Today Technol. 7: e157-e162.

SCU - Science Communication Unit, University of the West of England, Bristol (2013) Science for environment policy in-depth report: Soil contamination: Impacts on human health. Report produced for the European Commission DG Environment, September 2013. Available at: http://ec.europa.eu/science-environment-policy.

Shayler H, McBride M, Harrison E (2009) Sources and impacts of contaminants in soils. Cornell Waste Management Institution, NY, USA, 6 p.

Teuten EL, Saquing JM, Knappe DRU, Barlaz MA, Jonsson S, Björn A, Rowland SJ, Thompson RC, Galloway TS,
Yamashita R, Ochi D, Watanuki Y, Moore C, Viet PH, Tana TS, Prudente M, Boonyatumanond R, Zakaria MP, Akkhavong K, Ogata Y, Hirai H, Iwasa S, Mizukawa K, Hagino Y, Imamura A, Saha M, Takada H (2009) Transport and release of chemicals from plastics to the environment and to wildlife. Philos. Trans. R. Soc. Lond. B Biol. Sci. 364: 2027-2045.

Trott O, Olson AJ (2010) AutoDock Vina: improving the speed and accuracy of docking with a new scoring function, efficient optimization, and multithreading. J. Comput. Chem. 31(2): 455-461.

Valentın L, Nousiainen A, Mikkonen A (2013) Introduction to organic contaminants in soil: Concepts and risks. In Emerging organic contaminants in sludges: Analysis, fate and biological treatment, Vicent $\mathrm{T}$, Caminal $\mathrm{G}$, Eljarrat E, Barceló D (eds.), Springer-Verlag Berlin Heidelberg, p. 1-29.

WHO - World Health Organization (2013) Contaminated sites and health. Report of two WHO workshops; Syracuse, Italy, 18 November 2011 and Catania, Italy, 21-11 June 2012, 106 p.

Yao Y, Sack TU, Volchek K, Brown CE (2015) PFCcontaminated soil and its remediation strategies: A review. Proceedings of the Thirty-Eight AMOP Technical Seminar, Environment Canada, Ottawa, ON, p. 314-339. 